passed away due to primary disease progression. The mainstay of our analysis was to look at factors associated with delay in decannulation among children and the associated morbidity.

\section{P185 DEVELOPMENT OF A BRONCHIOLITIS ASSESSMENT TOOL IN TWO BUSY UK DISTRICT GENERAL HOSPITALS: 'BRONCHIOLITIS-MADE-EASY'}

1,2Kene Maduemem* ${ }^{1,2}$ Dilip Vasudeva, ${ }^{2}$ David Broodbank, ${ }^{1}$ Amol Chingale. ${ }^{1}$ Lincoln County Hospital, Lincoln, UK; ${ }^{2}$ Pilgrim Hospital, Boston, UK

\subsection{6/archdischild-2019-epa.540}

Background/Aim Bronchiolitis is one of the commonest causes of hospital presentations during the winter period. It poses a great deal of strain on the health care staff and resources. A targeted assessment tool is thought to provide a prompt and optimal assessment to limit unwarranted investigations and hospital admissions. The acute assessment of children with a clinical diagnosis of bronchiolitis was initially audited with subsequent re-audit using the assessment pro forma.

Methods Children admitted with bronchiolitis between November 2017 and January 2018 in one hospital site were randomly selected. Medical notes were reviewed to evaluate the clinical assessments and treatment(s) offered in comparison with NICE guidance. A pilot study was subsequently performed to assess the use of the new assessment pro forma by medical students and junior doctors.

Results Thirty medical notes were randomly selected for the audit. History of apnoea was inquired in 7 of 20 infants aged 3 months and below. Oral intake was clearly documented in only $36.7 \%(11 / 30)$. Neonatal events were clearly documented in $96.7 \%$ (29/30). Documentation of exposure to passive smoking occurred in 23.3\% (7/30). Only 50\% had documented assessment of hydration status and femoral pulses. Blood tests were requested in 13 infants with clinical indications documented in 7. Sixty-five percent (11/17) had no documented clinical indication for chest radiograph. Inappropriate treatments (bronchodilators, steroids, antibiotics) were initiated in $30 \%$ of infants. The pilot study involved 15 volunteers (medical students and junior doctors) on the use of the assessment pro forma in the 2 hospital sites. Analyses of the pro forma demonstrated $100 \%$ compliance in all parameters re-audited.

Conclusion The new pro forma has demonstrated its usefulness in targeted assessment of children with suspected bronchiolitis. Well-focused history and examination will safely stratify children to be managed in the community or hospital. The feedbacks received from its use were excellent.

\section{P186 LYMPHADENOPATHY REFERRALS TO A GENERAL PAEDIATRIC DEPARTMENT}

Petra Buckley*, Jean Donnelly, Suzanne Kelleher. OLCHC, Dublin, Ireland

\subsection{6/archdischild-2019-epa.541}

The aim of the study The aim of the audit was to determine if patients referred with lymphadenopathy need to wait for a General Paediatric outpatient appointment or if instead they could be reassured with normal investigations prior to attending the clinic, with the aim of providing a guideline for community management in some cases.
Method Lymphadenopathy is a common cause of presentation at GPs and a cause of concern for parents. We analysed a number of tertiary referrals to the General Paediatric Department in OLCHC to determine what and if any investigations should be performed for these patients and how we might improve the patient experience for these cases. Between September 2017 and January 2019, General Paediatric Triage Clinic received 12 referrals of patients with lymphadenopathy from GPs. Less than 50\% of referral letters included information about the size, mobility, consistency or tenderness of the concerning lymphatic nodule. None of the referral letters included blood test results or any other investigations. All referrals were asked to attend for blood tests including FBC, $\mathrm{U}+\mathrm{E}$, CRP, Urate, LDH. Depending on the results, further investigations were ordered or patients were discharged.

Results 8 (66\%) patients attended phlebotomy for blood tests. $50 \%$ of blood tests performed were normal, of these 2 patients were discharged from Triage Clinic and 2 patients remained on waiting list. 4 patients had abnormal blood results, 3 had a neck ultrasound performed, all of which were normal. 2 were subsequently discharged from Triage Clinic, 1 remained on waiting list. 1 patient was seen in Paediatric OPD and was discharged based on reassuring clinical examination without any further investigations. 4 patients did not attend for blood tests, 3 were discharged from Triage Clinic after not responding to multiple notifications. 1 remains on waiting list awaiting response to second notification.

Recommendations Developing a clinical guideline for lymphadenopathy with clinical examination and investigation criteria warranting referral to General Paediatric clinic which will empower GPs to perform appropriate investigations including the ultrasound prior referring patients on, therefore reducing the waiting times in our outpatient clinic.

\section{P187 KEEPING CHILDREN SAFE FROM MEDICATION HARM: THE MEDSIQ JOURNEY}

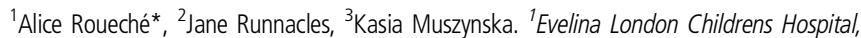
London, UK; ${ }^{2}$ Royal Free Hospital, London, UK; ${ }^{3}$ Royal College of Paediatrics and Child Health, London, UK

\subsection{6/archdischild-2019-epa.542}

Background In 2015 the UK Royal College of Paediatrics and Child Health (RCPCH) launched www.medsiq.org, an innovative website for sharing resources and quality improvement projects relating to paediatric medication safety. MedsIQ was founded in response to increasing awareness that children and young people are frequently harmed by errors in their medication. Children are 3 times more likely than adults to be harmed by medication errors and it has been shown that up to $15 \%$ of inpatient prescriptions have errors on them (1).

What is MedsIQ? Medication safety is a complex issue and innovative approaches to combat the problem are needed. Part of the answer lies with the medical community; most clinical teams have tried to tackle the problem; by auditing prescribing standards or having pharmacists on ward rounds, through to more innovative drug calculator apps, e-prescribing tools and Druggles (a safety huddle focusing on medication errors). The MedsIQ platform has been developed to allow people to share their ideas. Submitted projects and resources are peerreviewed and shared online. Alongside the website, there is a Champions' Network - a community of practice in improving paediatric medication safety, who participate in webinars and 